\title{
DICOM and XML usage for Multimedia Teleconsultation and for Reimbursement in Cardiology
}

\author{
N Balogh ${ }^{1}$, G Kerkovits $^{1}$, M Eichelberg $^{2}$, D Lemoine ${ }^{3}$, V Punys ${ }^{4}$ \\ 1. Semmelweis University CorpusNet foundation/MeData, Budapest , Hungary \\ 2. University of Oldenburg, Germany, 3. University of Rennes, France, 4. Technical University, Kaunas, Lithuania
}

\begin{abstract}
We've defined an open, scaleable telemedicine architecture to reduce the time of reports delivery and consultation; increasing their simplicity via introducing common image capture, compression, storage, presentation- and telecommunication formats and methods for telecardiology based on DICOM. We've developed a software application to implement them in 4 Hungarian and 4 Lithuanian cardiac clinics, introducing the most appropriate digital imaging methods and formats using the latest available technology.

We describe data and document exchange among health care providers (hospitals, GPs etc.) and the reimbursing insurance institutes introducing XML technology. We present the most important XML datasets for reimbursement in Hungary, the validation schemas and procedures, comparing the XML and old data file sizes with different compressions and (server and client) $X M L$ tools. The comparison includes different schema file sizes, and validation times.
\end{abstract}

\section{Methods and results}

We searched open, flexible solution for multimedia data exchange among healthcare provider institutions and for insurance data exchange between these institutions and the reimbursing insurance companies.

\subsection{Multimedia data exchange among healthcare provider institutions in cardiology}

For the definition of the content and format for multimedia reports and consultation we've used international literature and our own experiences and possibilities. In addition to textual and numeric data this includes still image and movie (loop) capture, storage, processing, presentation and communication for cardiac ultrasound and angiography. We've examined the necessary hardware, software and data transmission techniques and formats for different telecommunication speeds.

Angio machines produce direct digital output in DICOM format, so image capture and storage is easy.

Direct digital output (via port or disc) from ultrasound machines however is rare in our evaluation countries.

Ultrasound labs use SVHS VCR to record ultrasound video loops from ultrasound machines' SVHS port. SVHS/Hi8 gives 400 lines resolution. Frame grabbing from this SVHS output port and from VCR/camcorders we've compared MJPEG (Motion Joint Photographic Expert Group), miniDV(Digital Video) and MPEG2 (Motion Picture Expert Group) formats; capture-, processing- and storage technologies. Using full PAL (720x576 pixel) resolution and 25 frames per second we've examined different hardware and software tools comparing image quality, frame drops, raw data and final loop sizes, as well as the process easiness. Raw PAL video in 24 bit RGB mode and $25 \mathrm{fps}$ means $31 \mathrm{MB} / \mathrm{s}$ data. Using YCBCr 4:2:2 format this decreasing to $21 \mathrm{MB} / \mathrm{s}$.

MJPEG use this 4:2:2 format and we've used 5:1 compression during capture with $7 \mathrm{MB} / \mathrm{s}$ PCI frame grabber card (and 20MB/s U2W SCSI hard disk to capture AVI video).

PAL MiniDV use 4:2:0 format allowing 500 lines and $3,6 \mathrm{MB} / \mathrm{s}$ video data rate. We've used 3 methods to test DV with Digital8 camcorder, capturing loops from it with 1394 PC card to hard disk (in AVI format): record miniDV format on $\mathrm{Hi} 8$ video tape, "pass through" facility of the camcorder to record directly to hard disk (bypassing recording on tape), finally play analogue Hi8 tape on Digital8 camcorder to get DV output for PC.

Both MJPEG and DV using compression on each consecutive still images to decrease movie data size.

MPEG2 using compression in time; only the changes (to key frames) in consecutive image frames are stored. We've used MPEG2 PCI frame grabber card in PC to capture from the ultrasound machine or from the SVHS VCR or Hi8 camcorder. We've used 4, 8(DVD) and 10 $\mathrm{Mb} / \mathrm{s}$ (variable) bitrate during MPEG2 capture.

After our double blinde compression study (using nearly 500 loops by 8 cardiologists and 5 IT personnel), we've found, that differences in image quality are not substantial. Capturing directly from the port of the ultrasound machine gives slightly better results than from tapes, but more inconvenient. DV is less resource demanding than MJPEG, still it gives better quality because less compression is needed (4:2:0) and because of the more intelligent nature of DV codecs. There was no frame drop in MPEG2 and we've got less frame drops in DV than in MJPEG capture. Both MJPEG and DV need large disk space, but framing is very accurate -if the lab has time to cut the loops. We can convert the cut mini DV loops to MPEG2 for less space, but this is usually 
software only solution (with additional step in the process) and very much depend on PC processor type and speed.

MPEG2 gives the smallest space and very good image quality using appropriate capture cards. DVD MPEG is more close to the original ultrasound screen (what we've used to) than DV (where contrasted lines appearing sometimes), especially on TV screen. Cut is less accurate though, so if the lab is too busy to cut the loops MPEG gives the best price/performance solution. Windows size is more easiliy resizeable for MPEG2 than for DV in Windows Media Player for quick review.

Full MPEG2 uncut ultrasound study takes 50-100 MBytes which is good for cheap CD recording. Full DV uncut ultrasound study takes 400-1300 MBytes for what DVD recording seems the best technical candidate (cut or "clinical compression" may decrase the sizes to half). Latest DVD-R/RAM\&DVD-R or DVD+RW\&CDR-W combo writer drives give good technical opportunity. Using 1 cartridge for several patients, Hi8 video tape with Digital $8 \mathrm{DV}$ format is a cheap digital solution for mid term storage (as it is magnetic and not optical).

There is no common optimal solution both for telereporting and teleconsultation.

We've defined an architecture called SAMTA (Scaleable Architecture for Medical multimedia Telecommunication Applications) and have developed an application software based on that:

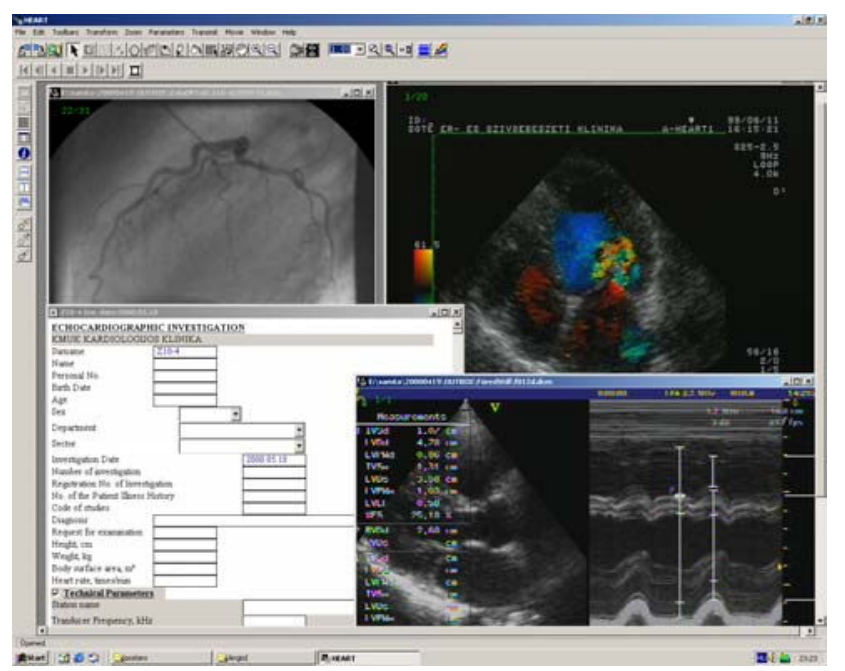

Figure 1 The image processing reporting and communication feature of SAMTA application

With this application we can write reports, digitize ultrasound movies directly from the machine's SVHS port (in MJPEG or MPEG2 format), read DICOM Angio CDs, cut and store these clips, process them (e.g. scaleable compression) and communicate them among 4 Hungarian and 4 Lithuanian cardiac centres for telereporting and teleconsultancy using DICOM3. We're using and have evaluated JPEG, MJPEG, MPEG2 and our own compression techniques.

We've further processed these clips converting them to DICOM3 with our own developed software package. In our package we use image segmentation technique to reduce ultrasound loop sizes with thirty percent:

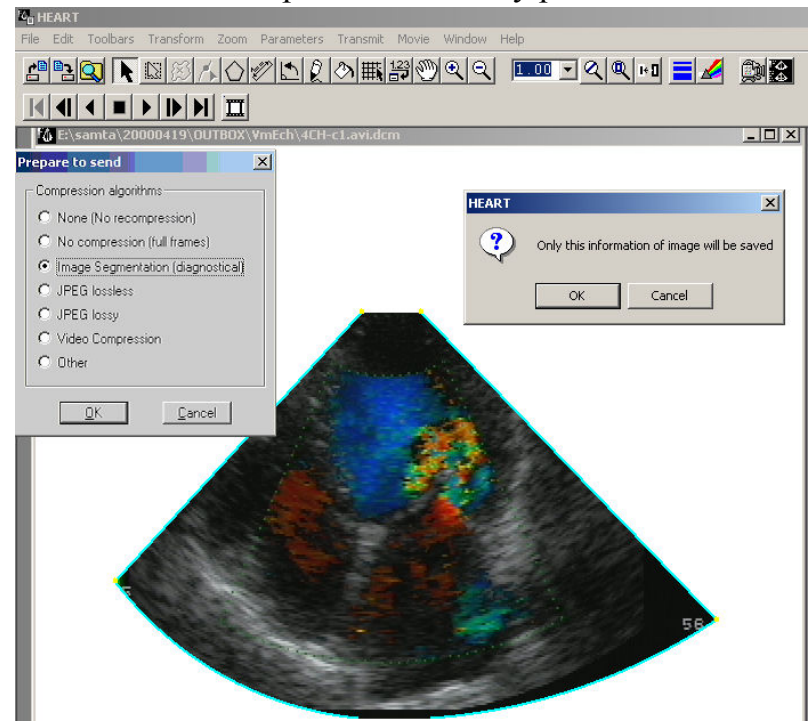

Figure 2. Image segmentation in SAMTA application

For DV and MPEG2 we've used third party nonlinear editing software packages.

As patient moves among health institutions his/her data should move too. Test requests and results, prescribings, discharge reports should be exchanged online.

Document (eg. Discharge report) and data exchange is not simple even inside hospital departments as the reports contain data fields from RDBMS-es (like lab data).

In SAMTA, developing the necessary templates we've used XML for textual and numeric data distribution (even over GSM line with Palmtops for emergency) and we have used SMIL for the synchronised authoring and play back of texts, sounds, images and movies for multimedia cardiac telereporting over dial-up, ISDN or leased lines. Most of our results can be used in local cardiac networks (PACS) inside the hospitals or in Intranets

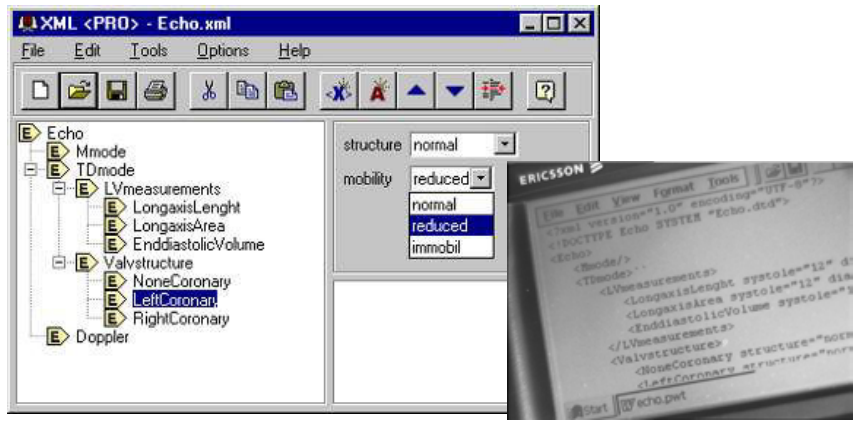

Figure 3. Part of Ultrasound test result XML file

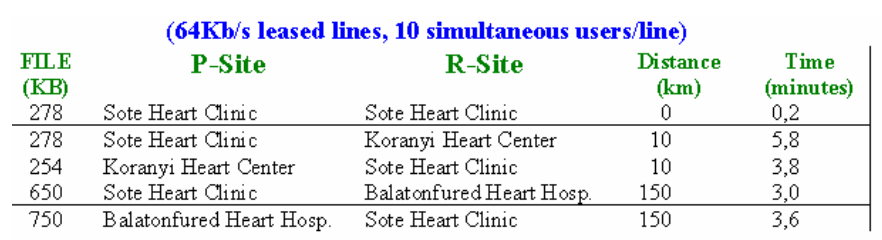

Table 1. Transfer times of different files among Hungarian Cardiac clinics from Patient (P) site to Remote (R) site. 


\subsection{Insurance data exchange between healthcare providers and insurance institutions}

We've examined how the present heterogenous exchange formats in Hungary (fix lenght records, DBF, Excel etc.) can be transferred to XML and how they can be validated on-line by providers to a schema provided by National Health Insurance Institute (NHII).

We've transferred and compared all datasets to XML for all kind of health providers, but as the most critical amount is the outpatient data produced by the University clinics we've examined that in more detailed. This data is sent in one package in fixed record lenght format and can be as large as 12 Mbytes for a clinic.

As can be seen in Figure 4, the size of XML is larger than present used ones (12MB grown to $44 \mathrm{MB})$. We can compress XML however quite well, depending on the content. For the outpatient data, the best result is given by algorithms using the speciality of XML tag syntax. This is followed by DOM1, WinZip and DOM2 respectively. As the original data files are sent usually in compressed way, so we need to compare the compressed data sets. Theoretically DOM compression is better, but there are practical issues (like market share and longevity etc.) as well.

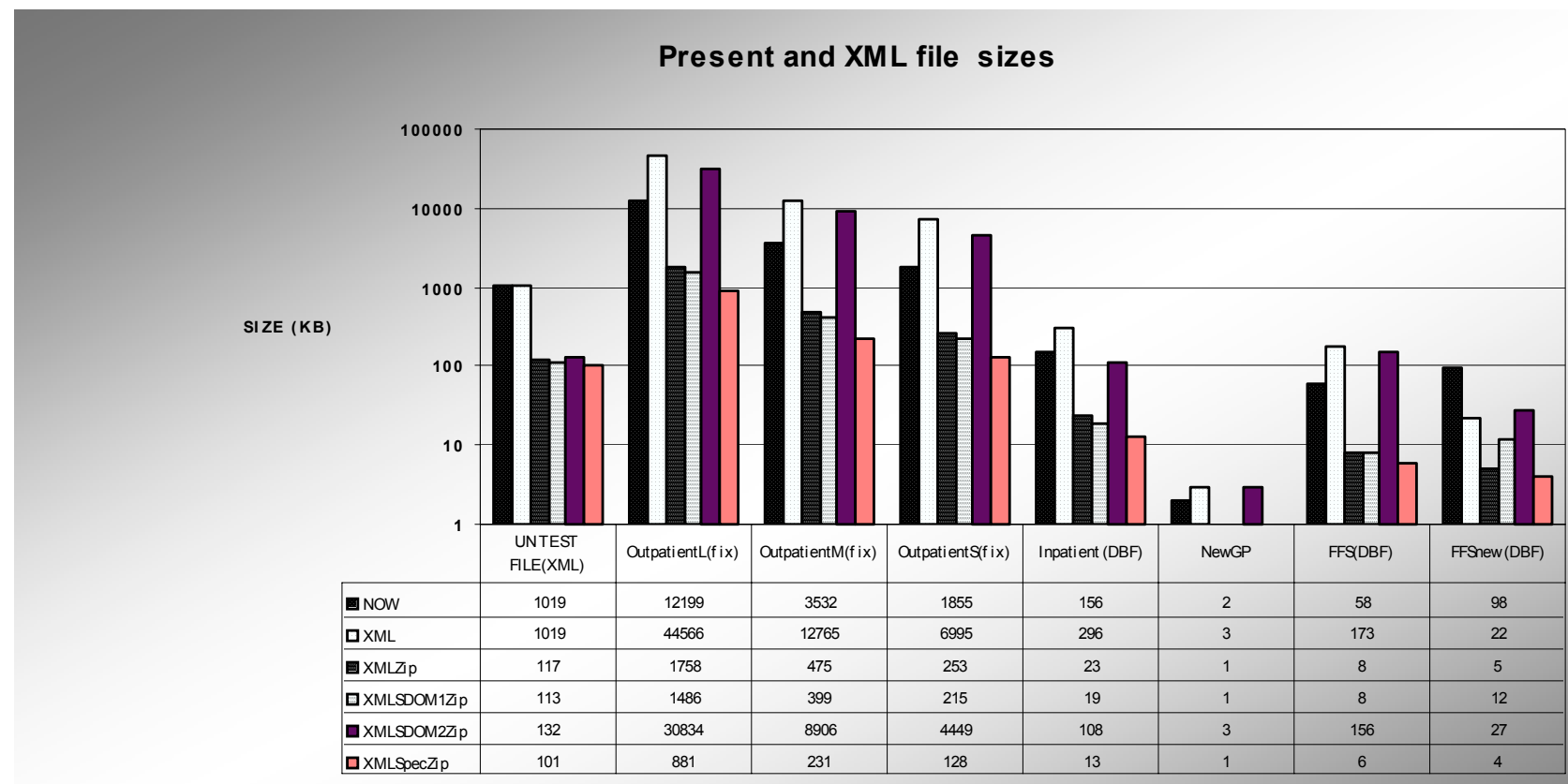

Figure 4. File sizes results of different XML compressions for different healthcare provider types in Hungary

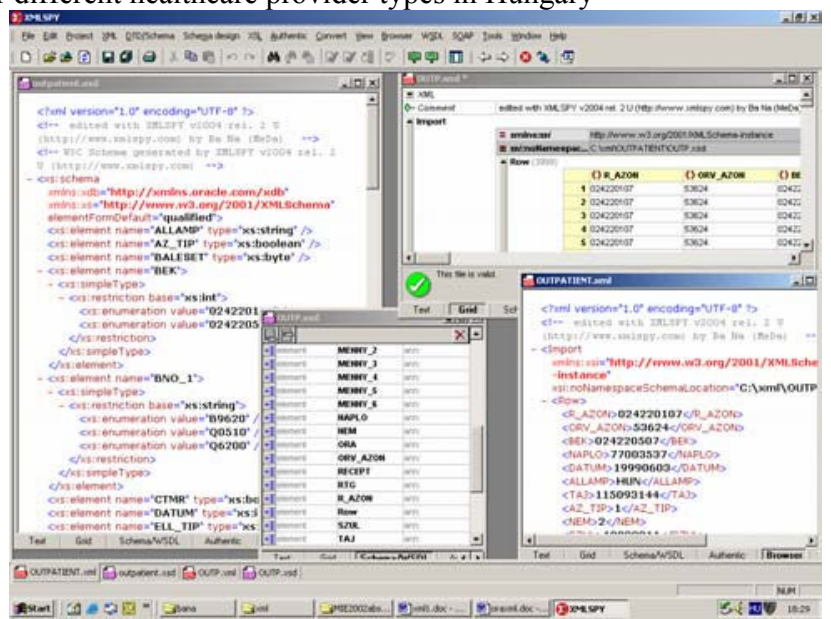

Figure 5. XML dataset validation process screenshot for Hungarian outpatient reimbursement dataset (as seen "The file is valid")

Based on the compressions above we can use XML even the original XML file sizes are larger (a 44Mbyte XML became 881 Kbyte using XMLspeciZip).
The time required for compression is not substantial, it was below 1 minutes even for the largest datasets.

\begin{tabular}{|l|l|l|l|l|l|l|l|l|l|l|l|l|}
\hline $\begin{array}{l}\text { Size of } \\
\text { XML } \\
\text { dataset }\end{array}$ & $\begin{array}{l}\text { Lo } \\
\text { ad }\end{array}$ & \multicolumn{6}{|l|}{ VALIDATION TIMES (SEC) } & AND & $\begin{array}{l}\text { Rep } \\
\text { lace }\end{array}$ & $\begin{array}{l}\text { Con } \\
\text { ver } \\
\text { sion }\end{array}$ \\
\hline & DTD & $\begin{array}{l}\text { Si } \\
\text { ze }\end{array}$ & $\begin{array}{l}\text { Biz } \\
\text { Talk }\end{array}$ & size & $\begin{array}{l}\text { W3 } \\
\text { C }\end{array}$ & $\begin{array}{l}\text { Si } \\
\text { ze }\end{array}$ & $\begin{array}{l}\text { XM } \\
\text { L } \\
\text { Data }\end{array}$ & $\begin{array}{l}\text { Si } \\
\text { ze }\end{array}$ & \\
\hline $\begin{array}{l}4000 \\
\text { records }\end{array}$ & $\begin{array}{l}120 \\
\text { sec }\end{array}$ & $\begin{array}{l}30 \\
\text { sec }\end{array}$ & $2 \mathrm{~K}$ & $\begin{array}{l}50 \\
\text { sec }\end{array}$ & $6 \mathrm{~K}$ & $\begin{array}{l}25 \\
\text { sec }\end{array}$ & $4 \mathrm{~K}$ & $\begin{array}{l}40 \\
\text { sec }\end{array}$ & $\begin{array}{l}6 \mathrm{~K} \\
180 \\
\text { sec }\end{array}$ & $\begin{array}{l}60 \\
\text { sec }\end{array}$ \\
\hline $\begin{array}{l}65527 \\
\text { records }\end{array}$ & $\begin{array}{l}\text { Too } \\
\text { hs }\end{array}$ & muc & h wa & itin & g ti & mes & & & $\begin{array}{l}2 \\
\text { hs }\end{array}$ & $\begin{array}{l}2 \\
\text { hs }\end{array}$ \\
\hline
\end{tabular}

Table 2. Times required for XML validation and other processes for different XML data sizes

Validation times at client side are too long for 65 thousands records. Validation however can be done at server site or separately by the different departments of a large outpatient clinic (to get the above 4 thousands records).

The conversions to/from XML might be annoying in routine use. Latest RDBMS-es (like Oracle XML DB) support XML in "native form", that means once the 
necessary prework is done with the best options, the XML data can be used similar to relational data:

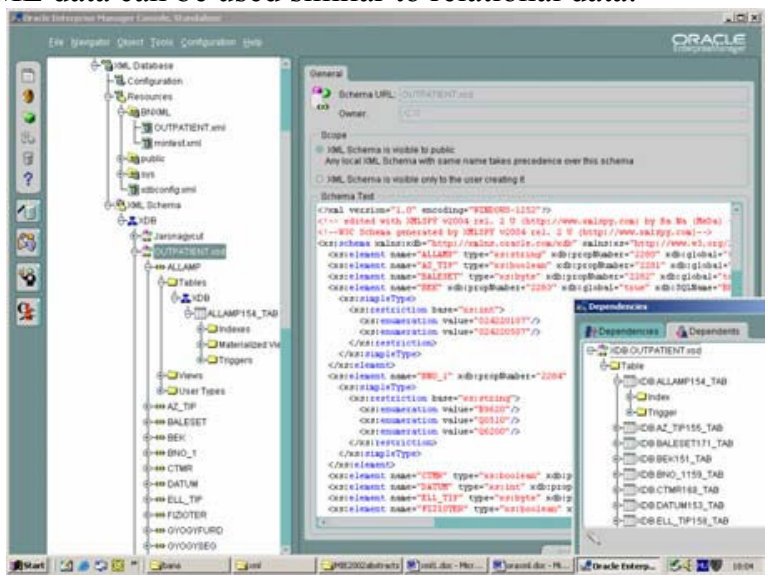

Figure 6. Registered outpatient XML Schema and Resource files in traditional RDBMS.

It is not easy to separate the XML data (so to know precisely its size for comparison), as the relating XML Database file contains both the schema and the dataset as seen above for the outpatient dataset for NHII (resources and schemas).

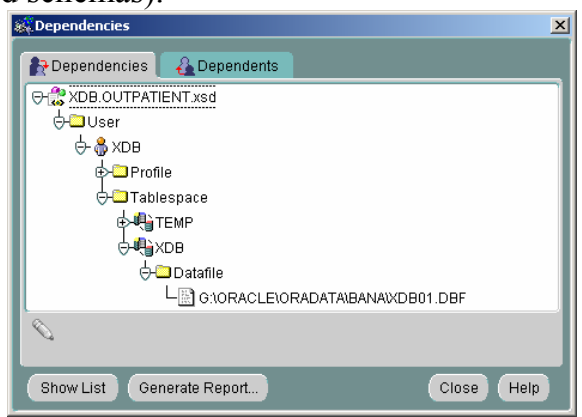

Figure 7. XML physical data file path in RDBMS.

We can see the size of that file compared with the original XML flat file:

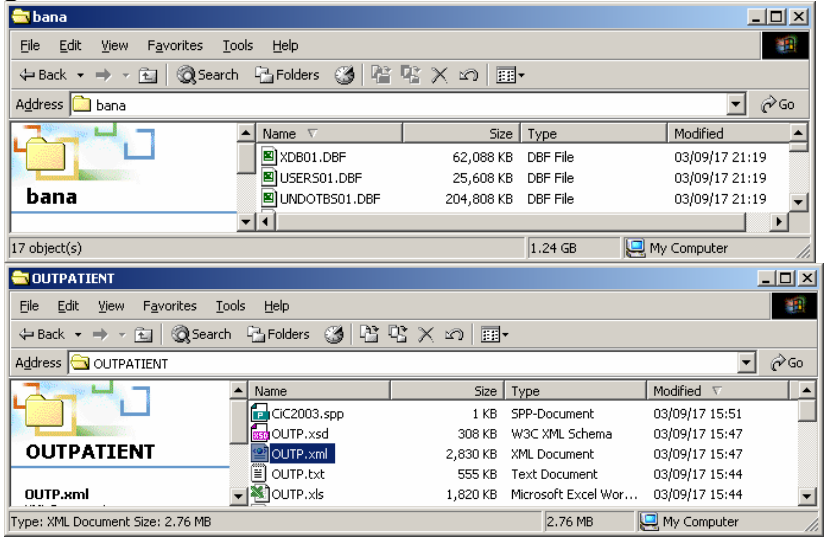

Figure 8. File sizes for outpatient XML Dataset in RDBMS and in flat files.

The XML instance documents saved in the database must conform to an XML Schema. First we need to register XML Schema (eg. W3C as seen above) for wich XML Database provides a mechanism. If we've prepared an XML schema that is annotated with information to automatically generate object datatypes and object tables, the database automatically generates these objects and we can insert conforming XML documents into it. Then we can view the elements in their XML form. We can also view the dependent objects such as tables, views, indexes, object types, array types and table types. If we issue a query in SQL scratchpad, we can view the instance data stored in the database corresponding to that element. Registering schemas and adding resources to XML Database might be time consuming (at least with the beta versions of the supporting XML DB), depending on the sizes of them (even for the 4 thousand records of our outpatient datasets above). We need to do it only once though.

\section{Conclusions}

If ultrasound machines had DV output (beside SVHS) that would help precise digital imaging a lot. Without that, DVD MPEG2 or 4 (with 6-8Mbps bitrate) will play serious role because of it's less demanding PC infrastructure and strong support from movie industry (that means cheap capture, storage and playback). Using SAMTA, reporting and consultation are more simple and unified. Concerning the amount of data for loops, and the low bandwith of telecommunication channels in Central European countries, we have to use off-line preprocessing, incorporating "clinical" and lossy technical compression techniques (4-12:1 for angiography and 6-18:1 for ultrasound).

XML has large technical potential for data exchange among healthcare and reimbursing institutions. The difficulty is the content represented by schema and tag definitions. We can use SNOMED or National Library of Medicine (USA) definition of terms to define Hungarian tags. This job can be done in the framework of different medical societies and advisory bodies using the appropriate motivation for their members.

\section{References}

[1] Nandor Balogh, Gabor Kerkovits, Marco Eichelberg, Didier Lemoines, Vytenys Punys. Cardiac multimedia reports on the internet using DICOM. 2000, ISBN-0-444-50536-9, pp.1032

[2] Nandor Balogh. The role of XML in medical informatics in Hungary. 2002, ISBN 158603279 8, p 168-173.

\section{Address for correspondence:}

Name: Dr. Nandor BALOGH

Full postal address: Ratkoc u. 9. Budapest, Hungary H-1118

E-mail address: balogh.nandor@iif.hu 